\title{
The HELP System
}

\author{
T. A. Pryor, R. M. Gardner, P. D. Clayton, and \\ H. R. Warner
}

Development of a comprehensive computer system for acquiring medical data and implementing medical decision logic has been ongoing for over 15 years at the University of Utah and the LDS Hospital in Salt Lake City, Utah. This system is known as HELP and is currently operational at LDS Hospital, which is a 550-bed tertiary care hospital serving the needs of the intermountain west. This hospital also serves as one of the primary teaching centers for the University of Utah Medical School. Having been developed in this environment, the design of the HELP system was required to meet the administrative, clinical, teaching, and research needs of hospitals, as well as provide the decision-making capability.

\section{SYSTEM OBJECTIVES}

Four major objectives were outlined in the development of HELP. They are as follows. (1) The system must be able to accommodate an ever-expanding medical data base. That is, the system must easily allow for expanded definition of new medical terms within the internal HELP data base. To facilitate this objective, the system had to provide generalized procedures for the acquisition, storage, and review of all data items defined in the medical data base. (2) The system must process medical decision logic. The decision logic supported by HELP had to include criteria for diagnostic, therapeutic, and alarm protocols. (3) The system must be capable of serving both the medical and administrative needs of the hospital. HELP, therefore, was designed to be a complete hospital information system. (4) The system must provide effective research subsystems that facilitate clinical research on the large data base naturally acquired by the system.

Of the above objectives, the most unusual and potentially most beneficial is that feature which implements medical decision logic. In the development of this capability, certain design constraints were formulated: (1) The decision logic should be modular, with control and interaction of modules determined by medical experts responsible for a

This paper was presented at the Sixth Annual Symposium on Computer Applications in Medical Care, held in Washington, D.C., October 30-November 2, 1982, and is republished here with the permission of SCAMC and IEEE. (C) 1982 IEEE.

From the Department of Medical Biophysics and Computing, University of Utah, Salt Lake City, Utah. 
given module. Each decision module should be readily added to, modified, deleted, and understood by physicians and other members of the medical community not especially trained in computer science. This constraint led to the development of a HELP language and programs to write HELP protocols. These programs for developing medical logic were designed to be "user-friendly," which allows non-computer-science-trained individuals to interact with the HELP protocols after minimal training. The key feature of the HELP language has been the ability to define precise search criteria for patient data within appropriate time windows. Once the appropriate patient data search statements have been written in HELP, the language provides for either deterministic or statistical logic to be easily applied on those data items retrieved to complete the decision criteria. (2) The processing of decision criteria should be data-driven. That is, the decision criteria, once verified by their author, are automatically processed whenever data are acquired that are referenced in a logic sector. For example, if a HELP protocol is written to alarm on certain electrolyte abnormabities, that HELP protocol should automatically be evaluated for a patient whenever clinical laboratory electrolyte data are stored in the patient's medical record. This feature of HELP means that medical decision criteria are evaluated in minimal physician interaction within a timely fashion and conveniently presented to the physician. The physician is not required to enter the data or request evaluation of the decision logic from a terminal before receiving the results of such evaluation. He need only review patient information from either the terminal or hard copy reports. One of the more important pieces of data that can be used to drive the decision protocols is time. Because of this feature, HELP protocols may be written that may be used for hospital-wide patient monitoring at set periodic intervals. For example, in monitoring potassium levels, a decision may be written to notify the doctor of the need to order the appropriate laboratory test. Then, for a patient who is being administered a potassiumsparing diuretic and perhaps a potassium supplement concurrently, the HELP system can check preset intervals to see if an electrolyte packet is ordered. If not, the physician can be alerted to the need to order such a test to properly monitor the electrolyte levels in the presence of the prescribed drug. (3) The system has been designed to provide output to a variety of sources. Thus, the designer of HELP protocols may transmit the results of the HELP protocols to several destinations, including the patient's medical record, the printers located at nursing divisions, the departments performing specific tests, or special research files set up by the user for other purposes. (4) The system has been designed to interact with the data-acquisition modules of HELP ("ask mode"). This query feature has been added to allow HELP protocols that require certain items of medical data to be present before processing of that module may be completed. After evaluating an appropriate search statement to verify whether or not the data are present, the decision logic may, if the data are not present, instruct the "query" module of the HELP system to alert the appropriate person that specific information should be entered into the system. The information to be entered may refer to a historical or physical finding, which would be directly entered through a computer terminal by either patient, nurse, or physician, or it may be a request to order a specific laboratory test. This feature has particular importance in allowing the system to minimize data entry from the physician or nuse since only data necessary to continue evaluation of specific hypotheses will be requested. This feature eliminates the shotgun approach in which all possible data must be entered by the medical personnel before activation of any decision logic. 


\section{HISTORY OF HELP}

The HELP system is the outgrowth of many years of continued development in making the computer a useful tool in the practice of medicine. It had its earliest beginnings in the late 1950s and early 1960s, when Dr. Homer R. Warner and associates at LDS Hospital began exploring the use of computers in the diagnosis of congenital heart disease. These early efforts culminated in the creation by the University of Utah of the Department of Biophysics and Bioengineering in 1963. One of the major goals of this new department was to explore ways in which a computer could enhance the decision processes in the medical environment. The early efforts of the department were directed toward the processing of analogue signals from patients. A dual CDC 3300 computer system supported by both the National Institutes of Health and the National Center for Health Services Research was used to develop and implement automated heart catheterization laboratory programs, patient monitoring systems for monitoring both hemodynamic and electrocardiographic parameters, programs for automated interpretation of the electrocardiogram, and programs for capturing and reporting of laboratory values from the clinical laboratory. As this research continued, it became apparent that the integration of the output of these individual systems could be of greater importance than any of the individual systems by themselves. Therefore, beginning in about 1970, all clinical systems programs were modified to use a common integrated data base system. The design and development of this data base system was the initial step in formulation of the current HELP system. The data base was designed for continued enlargement and definition of medical terms as newer systems and data items were added to the HELP system.

With the common data base system in 1975 began the development of a language and a system for processing of medical decisions. This early work defined the requirements necessary to implement the varied medical decisions in a system of sufficient latitude for practical use in a medical environment. Because of the experience that had already been gained in medical computing, and noting the similar experience throughout the country, the early design criteria included the need for modularity and the need for data-driven processing in order to minimize the data entry required by the medical personnel.

By 1978 terminals had been placed in every nursing division in LDS Hospital, and all of the intensive care wards, surgical suites, and laboratories had been instrumented for data acquisition and reporting. It was apparent, however, that the hardware on which the system had been developed was now archaic and there was a need to redevelop and implement the system on modern "state of the art" computers. At this time, the LDS Hospital was also developing plans to meet the administrative needs of the hospital and felt that any subsequent computing system installed in the hospital must satisfy those needs, as well as the clinical needs that had been serviced by the HELP system. Therefore, in the conversion and redevelopment of the HELP system, the design criteria were enlarged to include not only the medical goals previously stated but also the goal of serving the administrative needs of the hospital. A Tandem computer system was chosen as the most logical one for development of the new HELP system, since it provided the capability for redundancy and minimal downtime plus the ability to grow easily to meet increasing needs on the system. The conversion process began in February of 1979. The 
transfer of administrative and clinical services to the Tandem was initiated in October 1980 , with all systems being converted by August of 1981 . This conversion included all of the previous clinical functions that had been running on the Control Data equipment together with the newer administrative programs.

The current system consists of a 6-CPU Tandem system with 770 million bytes of disk storage. They are interfaced to the system with 196 terminals and 95 serial printers for entering and reporting at the various nursing stations, intensive care wards, and laboratories. Since the Tandem computer system as purchased has capability of receiving only digital data, all analogue processing for the system is performed using 14 Data General Nova $3 / 4$ 's, which are interfaced to the Tandem system. The terminals, however, connected to a Nova system, have the capability of both calling programs to process on the Nova analogue signals from transducers connected to the patient and calling any of the programs on the Tandem central computer system.

\section{OPERATIONAL ASPECTS OF THE HELP SYSTEM}

This section will describe specific applications and capabilities of the HELP system as currently implemented at LDS Hospital.

\section{Clinical Data Base}

The data base consists of two elements: a long-term abstract of demographic and clinical information likely to be useful if the patient is readmitted to the hospital, and a short-term comprehensive collection of all data gathered during the current hospital admission.

Table 1 lists the clinical data items that are part of the clinical data base for each individual patient as appropriate.

All data are stored in coded form (as opposed to free text) so that data can be retrieved and analyzed for use in research and decision logic. Hierarchal codes are defined using a system called PTXT, which is basically a computer-based dictionary. Using this program, a user defines the codes for a data item, as well as the associated text to be used for reports and terminal display, and key words associated with the item. Key words enable users who are constructing search items for decision logic sectors to easily specify the codes that should be sought.

For example, to construct a search sector to find all patients who had evidence of pneumothorax by chest radiograph, one would specify that X-ray data were desired then enter the key word pneu. In response to this entry, 21 different data items are displayed on the screen, ranging from pneumonia as a reason for performing an examination, to the procedure labeled pneumoencephalogram. When the term Radiographic Finding, Pneumothorax is selected from this list, the corresponding codes for this data item are automatically transferred to the search item logic for the HELP sector or report form. By omitting the specifier limiting the search to X-ray data, all data items defined as part of the clinical data base whose key words began with "pneu ... "' could be reviewed; in this case, 59 separate data items are currently displayed for possible selection. 
Table 1. Data Items That Are Currently Part of the Integrated Clinical Data Base

\begin{tabular}{|c|c|c|}
\hline \multicolumn{2}{|l|}{ Clinical laboratory } & $\begin{array}{l}\text { Chemistry, hematology, urinalysis, microbiology, tox- } \\
\text { icology }\end{array}$ \\
\hline \multicolumn{2}{|c|}{ Catheterization laboratory } & $\begin{array}{l}\text { Oxygen saturation, hemodynamic pressures, valve } \\
\text { areas and gradients, shunts, cardiac output, ventric- } \\
\text { ular wall motion }\end{array}$ \\
\hline \multicolumn{2}{|l|}{ Pulmonary laboratory } & $\begin{array}{l}\text { Blood gases, spirometry, plethysmograph, diffusing } \\
\text { capacity, total lung capacity }\end{array}$ \\
\hline $\begin{array}{l}\text { Coronary ICU } \\
\text { Shock trauma ICU } \\
\text { Neuro ICU } \\
\text { Respiratory ICU } \\
\text { Thoracic surgery ICU } \\
\text { Surgical ICU }\end{array}$ & $\begin{array}{r}12 \text { beds } \\
10 \text { beds } \\
6 \text { beds } \\
3 \text { beds } \\
14 \text { beds } \\
6 \text { beds }\end{array}$ & $\begin{array}{l}\text { Hemodynamic monitoring, ECG rhythm monitoring, } \\
\text { cardiac output, fluid balances, drug monitoring, nu- } \\
\text { tritional monitoring, nurses notes }\end{array}$ \\
\hline \multicolumn{2}{|l|}{ Pathology } & Biopsies \\
\hline \multicolumn{2}{|l|}{ Radiology } & $\mathrm{X}$-ray findings \\
\hline \multicolumn{2}{|l|}{ Pharmacy } & Medications, TPN administration \\
\hline \multicolumn{2}{|l|}{ Demographic } & $\begin{array}{l}\text { Allergies, synopsis of past hospital admissions, admit } \\
\text { diagnoses, discharge diagnosis }\end{array}$ \\
\hline \multicolumn{2}{|l|}{ Infectious disease } & Urinary catheter monitoring \\
\hline \multicolumn{2}{|l|}{ Surgery } & $\begin{array}{l}\text { Hemodynamic monitoring, anesthesiologist report, } \\
\text { surgical procedures }\end{array}$ \\
\hline \multicolumn{2}{|l|}{ Multiphasic Screening } & Height, weight, blood pressure, ECG, laboratory \\
\hline \multicolumn{2}{|l|}{ ECG } & Rhythm, morphology, and serial changes \\
\hline
\end{tabular}

The hierarchal structure of the data base is designed to optimize the speed with which data items can be stored or retrieved. The first level of the hierarchy defines the general data class with which the data item is associated. There are currently 47 defined data classes in the system - one data class for radiology findings, a different data class for pharmacy (medications and allergies), a third for ECG interpretations, etc. There are 5 different data classes for data from the clinical laboratory (chemistry, hematology, urinalysis, microbiology, and drug levels in fluids). The second level in the hierarchal code structure defines the type of data according to the type of format in which the data are stored in the actual record in the computer.

Type zero data are defined according to specific bits or words in a fixed-length string. A SMAC 20 result from the clinical laboratory used this type of format, and every word in the string represents a value of a particular chemistry value whose units are defined in the PTXT definition.

Type one data codes are preceded by a delimiter that defines the data item to follow. The delimiters specify field codes (general subcategories within the data class), nouns (specific items), adjectives associated with a noun, adverbs (items associated with an adjective), data class modifiers (a modifier that can modify any noun in the data class), field code modifiers (a modifier that can modify any noun in the data class), field code, 
and various length numeric values. For example, a "PA and lateral chest examination" is a specific noun within the field code of "chest examinations" in the "radiology" data class. "Chest pain" is a field code modifier that could be given as a reason for ordering any of the specific procedures (nouns) in the field code category of "chest examinations." SNOMED, SNOP, and ICD9 codes are all stored in the type one data format.

Type three data are the results of previous HELP decision logic that are stored as part of the patient record and give the data class, field code, HELP sector number, value of the sector if appropriate, and any appropriate HELP sector modifiers (mild, moderate, severe, etc.).

The data are entered automatically in most instances through digital or analogue interfaces (ECG, catherization laboratory, pulmonary function laboratory, and clinical laboratory) or by interaction with a terminal (pharmacy, nurses' notes).

A general question-asking program (GQAP) makes it possible for technical personnel without programming skills to construct questionnaires. To develop a new questionnaire for data entry it is necessary to type a free text question, specify the type of answer expected, and define codes that should be associated with the answer so that GQAP can store the entered data in the patient record. Follow-up questions (depending on the previous answers) and default error-detecting logic are built into the system and are routinely used to construct branching-type questionnaires for data acquisition.

The data base generated while the patient is in the hospital is generally complete except for physician-derived data such as a complete history and physical finding. Research is currently under way to develop ways in which the physician can easily enter these missing data. Because the data base is currently hospital-oriented, physician office visit information and postdischarge items are generally not available except in specific research areas.

\section{Computer-Based Medical Decision Logic}

The most outstanding aspect of the HELP system is the ability to allow construction of modular sectors that may be used in the medical decision-making process. Sector logic can consist of straightforward deterministic "if . . . then. . . " rules or probabilistic applications based upon the Bayes formula. Sectors can specify a single modular decision, can call additional sectors, or can be nested to form a decision tree that gives patient-specific results.

HELP allows a user to specify the criteria for a medical decision. These criteria are compiled into decision logic sectors, which are stored on disk and activated by specific instruction or whenever data referenced by the decision logic are added to the data base.

Each HELP sector (set of criteria for a given decision or diagnosis) may consist of (1) a message or title that is displayed when the sector criteria are satisfied, (2) instructions to search for and retrieve specified data items from the patient's computer-based medical record, (3) a list of logical and/or arithmetic statements, (4) Bayesian probability calculations, and (5) references to other HELP sector evaluations.

To illustrate how sectors may be written, an example of a sector to predict the presence of pleural fluid by radiograph will be explained (see Figure 1). This sector is activated whenever a chest $\mathrm{X}$ ray is ordered for a patient. 


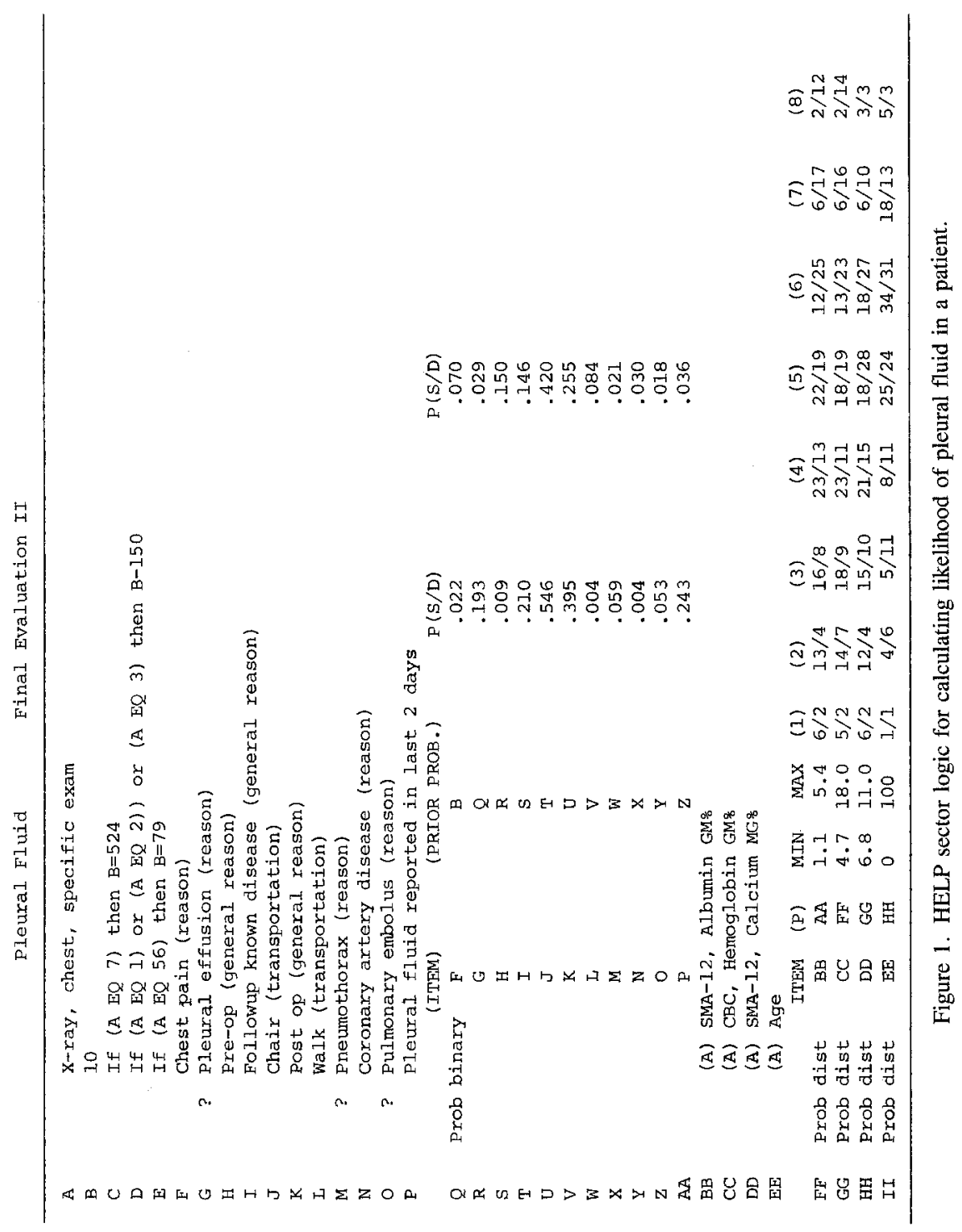


Item A causes the computer to search through the patient data base for an ordered chest examination that has not yet been processed by HELP. If such a data string exists, the value of item $A$ is set to a numeric code between 1 and 8 , according to the specific type of examination that was ordered. Item $B$ sets the initial a priori probability of having pleural fluid (for an inpatient at LDS Hospital for whom a chest $\mathrm{X}$ ray is ordered) at $1 \%$ $(10 / 1,000)$. Items $C$ through $E$ change the initial probability (value of item $B$ ) according to the specific procedure (value of item $A$ ) that was ordered.

After the a priori probability of pleural fluid is set according to the type of examination to be performed, the patient record is searched to retrieve other data that affect the likelihood of pleural fluid. Items $\mathrm{F}$ through $\mathrm{O}$ search for specific reasons for ordering the examination, special handling, and transportation. These items are used because analysis of the clinical data base showed that they significantly alter the likelihood of pleural fluid. The values for Items $\mathrm{F}$ through $\mathrm{O}$ are set to 1 (true) or 0 (false), depending on whether or not the code for that item is present in the data string that was created when the examination was ordered. These values are used in subsequent probability calculations. Item $\mathrm{P}$ is set to 1 (true) if the patient record contains X-ray examinations that showed pleural fluid within the last 2 days.

Items Q through AA perform binary Bayesian probability calculations based upon the data specified in items F through P. Bayes's formula is an algebraic expression used to calculate conditional probability.

Once the appropriate probability values have been obtained, application of the Bayes formula is straightforward in the binary case, i.e., when the symptom is present or not present. In item $Q$, the numbers .022 and .070 reflect the probabilities that, for an inpatient at LDS Hospital with and without pleural fluid by $X$ ray, chest pain (item $F=$ 1) would be given as the reason for ordering the $X$ ray. The value of item $B$ contains the prior probability to be used in the Bayes formula when item $Q$ is executed. In the instance of chest pain, the probability of pleural fluid is decreased if chest pain was given as a reason for ordering the examination. If the item upon which the calculation is based (in this case item F, chest pain) is not true, then the posterior probability is simply set to the prior probability. Item $\mathrm{R}$ then uses the result of item $\mathrm{Q}$ as the prior probability and calculates the conditional posterior probability according to whether pleural fluid was given as the reason for ordering the examination.

In the base of a continuous variable such as a laboratory value, the Bayes formula can still be applied using a distributed probability approach. In this instance the distribution of values of clinical parameter must be known for populations with the disease and without the disease. These probabilities can then be used in the same fashion as binary variables. To calculate distributed Bayesian probabilities using HELP, the parameter distributions are entered at the time the logic is constructed by dividing the distributions into bins and calculating for each bin the probability that patients from the diseased and nonaffected groups would have parameter values that fell into the range for that bin. The HELP logic uses the actual value of the clinical parameter to retrieve the appropriate probabilities for the calculation. Distributed Bayesian calculations are performed in items FF through II using the continuous variables listed in items BB through $\mathrm{EE}$. If they exist, the computer set items $\mathrm{BB}$ through $\mathrm{EE}$ equal to the most recent nonzero values for serum albumin (item BB), hemoglobin (item CC), and calcium (item DD), and age (item EE). Item FF calculates the probability of pleural fluid based upon the 
measured albumin value (item BB) for the patient using item AA as the prior probability. The format for the distributed Bayesian calculation specifies the range of possible albumin values (minimum of $1.1 \mathrm{~g} \%$ to a maximum of $5.4 \mathrm{~g} \%$ ) and, for eight bins, the probabilities that patients with and without pleural fluid would have albumin values within the bin. These are the eight pairs of numbers given as the last entries in item FF. Item GG is also a distributed probability calculation using the value of hemoglobin (item $\mathrm{CC}$ ) as the variable and the results of the calculation in item $\mathrm{FF}$ as the prior probability. If items $\mathrm{BB}, \mathrm{CC}, \mathrm{DD}$, or $\mathrm{EE}$ are not in the patient record, the appropriate (item value) is set to the prior probability for that line.

The final likelihood of pleural fluid is the value of line II and reflects the influence of laboratory tests, prior radiology findings, and reasons for ordering the examination. Only those data that significantly alter the probability are included in the HELP sector logic, and efforts are made to use parameters that are independent.

HELP sectors using similar types of logic have been written for a wide variety of applications. There are currently 2,147 operational sectors with clinical application. The most widely used sectors are those involved with ECG interpretation, blood gas interpretation, pharmacy alerts, predicted X-ray findings, and clinical laboratory alerts. The tools for constructing the medical logic are now operational, but the number of actual medical logic sectors must be regarded as only a beginning.

\section{Administrative Capabilities}

The core of the administrative functions is an admit-discharge-transfer (ADT) system. A patient can be admitted or preadmitted at several locations in the hospital (admitting, emergency room, radiology, multiphasic screening, clinical laboratory, outpatient area). After the patient's name is entered, the long-term file is searched for patients with similar names for verification and selection by the admitting clerk. If the address, insurance information, and other financial data are correct, the patient is simply admitted to the active short-term file. Otherwise, demographic and financial data are entered and the patient is admitted to the current and long-term file.

Preadmission scheduled patients and pending requests for room transfers generated on the nursing divisions are coordinated by the admitting office, which uses the computer to list which beds are expected to be vacated. As soon as a patient is moved to or from a bed, this fact is entered into the computer. Using the room-trace log generated for each patient and a list of standard room charges, the computer automatically generates room billing.

Ancillary test charges provide an example of the benefit of having an integrated financial and clinical data system. The tests are ordered through the nursing division terminals, and the "ready to bill" bit is automatically set whenever results are reported to the clinical data base. Charges for each test are obtained from the PTT file, which describes the code-associated text and standard cost for each procedure. Each department can make necessary changes to the standard charges for special cases. A transaction file exists to log-billable tests or supplies. By using alternate keys, each department can separately reconcile the day's procedures or an up-to-the-minute account may be obtained for a specific patient. Using programs to analyze the transaction file on a daily, weekly, or monthly basis, audit statistical summaries for departmental operations are generated. 
If the "ready to bill bit" is set, a program that is run at midnight of each day transfers charges to a separate financial billing computer. Via on-line handshaking a "billed" bit is then set in the status word of each entry in the transaction log for those procedures for which charges were transferred.

\section{Research Capabilities}

In order to analyze the resultant clinical and administrative data base, a query system called STRATO has been developed. The basic concept of this tool is that a HELP sector can be written to define the query criteria. The STRATO program then goes through the current patient file or predefined list of patients constituting a special study population to see which patients satisfy the criteria listed in the applicable HELP sector. Following this procedure one could, for example, obtain a population (list of patient numbers) of all male patients between 35 and 40 years of age who were admitted to the hospital and suspected appendicitis and received surgery. One could then write further HELP sectors that would define subpopulations of patients whose removed appendix was inflamed, who received certain antiinfective drugs, or who were treated by a specific physician. The STRATO program allows one to combine populations, obtain interations and unions of populations, etc.

Additional HELP sectors can be written to retrieve and compare variables (patient age, temperature before surgery, length of hospital stay, total hospital cost, e.g.) for alternative subpopulations. The variable or variables specified for each patient are stored on disk in a format related to the order of the patient number in the subpopulation. Statistical programs are interfaced to the STRATO system so that one can perform a regression analysis between different variables for the same subpopulation or compare the distributions (parametric or nonparametric tests) of the same variable across two or more subpopulations.

The results obtained from these searches can be used for administrative audits or research, or to obtain the conditional probability values needed in the Bayesian probability calculations of sectors written to make medical decisions.

In summary, STRATO can be used to derive populations or variables that are specified by the logic criteria expressed in specific HELP sectors. Such an approach gives the user ease, flexibility, and rapid results. The statistical packages provide additional tools necessary to analyze the data in a convenient fashion.

\section{EVALUATION OF HELP COMPUTER SYSTEM}

A survey conducted a few years ago by Battelle Laboratories of Columbus, Ohio, an independent evaluation group, revealed a positive attitude toward the use of the computer. As opposed to the usual negative attitudes and opposition encountered in adopting innovative things, especially in hospitals, a positive attitude toward the HELP computer system is prevalent among physicians on the staff of LDS Hospital. A second major finding of the Battelle study was that the highest proportion of physicians that were very enthusiastic about the system were specialists in surgery and internal medicine. Other specialists have not had as much use of the system. This study evaluated the 
expectations and utilization of the HELP system by response to questionnaires and also by individual interviews of key staff physicians. The overwhelmingly favorable response in terms of what physicians expected and received from the system encouraged us to further develop the system's decision-making capabilities.

The intensive and critical care units of the hospital have been computerized longer than most other areas of the hospital, so evaluation of physician and nurse responses might be representative of what other nursing areas and clinical areas of the hospital feel after they have had several more years of experience. A report from intensive care physicians as to what they felt were the advantages of the system will give some insight into the system's benefits. These physicians felt (1) that computer data are better organized and therefore more easily reviewed for clinical decision making; (2) that automated computerized decision making provides valuable data interpretation and alarming functions; (3) that the computer-generated reports and alarms focuses attention of the personnel on the treatment and management of most of the problems of the critically ill patient; (4) that the computer's ability to manipulate and calculate on quantitative data presents patient data and parameters in a form that enhances patient care; (5) that the computer accelerates the availability of data because of the on-line communications network interconnecting; (6) that computerized decision making provides an excellent basis for teaching logical decision-making skills to house staff and staff physicians; (7) that the quantitative structure provided by the computer system causes our physicians to think more quantitatively and, as a consequence, provides an excellent education tool in teaching quantitative and logical medicine; (8) that having all of the patient data in the computerized data base provides several advantages - it eliminates redundant charting, captures charges for clinical data entry, allows transfer of patients anywhere in the hospital without a loss of data continuity or of access, and, finally, allows data review on an ICU patient from multiple locations.

Evaluation from the ICU nurses of the system showed it to be of advantage in the care of the critically ill patient in the following areas. (1) All the laboratory data from the clinical laboratory is quickly available for review and, where desired, a hard copy printout can be generated. These data are available in a legible form as soon as the test is completed in the laboratory. (2) Blood gas analyses are performed in the pulmonary function laboratory, where analysis, computation, and interpretation by the computer, as well as prompt feedback of the results, provide a valuable tool in the management of especially-ventilator patients in the intensive care units. (3) The pharmacy module, coupled with the charting function outlined below, provides a major advantage to our clinical nursing staff. First, all drug interactions are monitored by the computer, since each drug order is entered into the computer by the pharmacist before the drug is dispensed. Drug-drug interactions, drug contrainteractions because of laboratory data, allergies, and other factors are evaluated on each patient, which minimizes drug-ordering errors. The computer generates a medication schedule for each patient at the beginning of each shift, which provides an easy method for checking what medications are to be given and when they are to be given. All of the drug-given information is logged into the computer. This drug logging not only becomes the official record for the patient but also is used to generate patient charges. The ability to handle drugs and intravenous fluid infusions with the system provides a tremendous advantage for the nurse in keeping track of fluid balance totals as well as charging for the solutions and drugs. Thus, once the 
nurse has completed the task of a computerized charting, all else that happens to the record is automatic. (4) The charting function is a very important function for nurses. Currently, the system generates 12-hour "shifts" and 7-day reports. These reports are extremely valuable tools not only for the nursing but for the medical staff in care of the patient. (5) The fluid intake and output summary provides valuable data as to how many units of blood and what other types of fluid intake and output the patient had during a particular shift or during a particular day. (6) Nutritional summaries are made available. These are felt to be an extremely valuable tool for both the nurse and the physician. (7) Interpretations of hemodynamic profiles that calculate parameters from cardiac output and pressure measurements are valuable.

The pharmacy module, which has been in operation since 1975, has had widespread acceptance by the medical staff and the pharmacy. Since the hospital is on a unit/dose system and since the computer provides the surveillance needed, we have been able to decentralize the pharmacy by using movable carts for the pharmacist and placing them with a clinical pharmacist on the nursing division. Having the pharmacist on the nursing division provides several advantages, both for the pharmacist and for the nursing and medical staff. The pharmacist is on the scene; if there are drug questions or problems, he can interact with the medical staff or nursing staff. If there are alerts that come up, these alerts can be quickly followed up with the medical staff. The one-to-one personal communication has dramatically improved the level of communication on drug problems as well as the ability of the nurse and the medical staff to order and dispense medication. Ongoing evaluations of alerts from the pharmacy module are performed by review of monthly logs. Table 2 summarizes the monthly logs of drug alerts generated by the system for 1 year.

The importance of having the computer perform the alert function is clearly demonstrated by the fact that only about $5 \%$ of the patients in the hospital have an alert; therefore, there is a large amount of uninteresting data that must be reviewed to get at those few patients and even fewer drugs ordered that represent problems. Those alerts that are truly life-threatening and that are not informational or have some subjective reason to be concerned about represent an even smaller fraction of the total alerts. Those alerts that require some physician interaction or changing of a drug or procedure are only about $20 \%$ of all of the alerts. That is, approximately $1.8 \%$ of the patients receive alerts that are truly life-threatening. This is considerably lower than reported by other studies, which further points out the need to have the computer perform this task. We have now

Table 2. Pharmacy Alerting Program

\begin{tabular}{lcc}
\hline & \multicolumn{1}{c}{$\begin{array}{c}\text { Monthly averages, } \\
\text { July 1981 to June 1982 }\end{array}$} \\
\hline Patients & 2,684 & \\
Patients generating & 227 & $8.45 \%$ \\
$\quad$ an alert & 49 & $1.8 \%$ \\
Life-threatening & & \\
$\quad$ alerts & & $94 \%$ \\
Physician compliance to & & \\
$\quad$ life-threatening alerts & & \\
\hline
\end{tabular}


a $94 \%$ compliance rate for those truly action-oriented life-threatening alerts. Therefore, approximately 50 patients each month are helped in some dramatic way by having the computer monitor the drugs ordered. The pharmacy module has resulted in an overwhelming positive response from the medical staff who make few medication-prescribing errors. The computer allows them to conduct their practice with the assurance that the problems will not be a major part of their risk in caring for the patient.

The X-ray decision-making area has also provided some unique advantages. We are currently utilizing the system on a daily basis not only to generate the orders and billing but also to capture the clinical results. At present we have 794 coded examinations, 435 reasons for performing the examinations, and 378 coded findings. Currently, about $62 \%$ of all examination results are completely coded and entered into the computer; that is, they require no additional explanatory information to be useful to the medical staff. Using the unique ability of the system to predict from a certain type of examination how the results might turn out, we are then able to generate the results in a very simple and compact form. Approximately $82 \%$ of the examinations are now reported merely by checking a box on the computer-generated report form. This not only is a great advantage to the radiologists who are reading the $\mathrm{X}$ ray but also is an advantage to the physicians who are caring for the patients since they can quickly review the information on the computer terminal. The radiologist interpretation data are then available after the report is completed by the radiologist.

An example of one of the specialized laboratories where the computer has been of great assistance is the pulmonary function laboratory. During the past year more than 62,000 blood gases and over 1,200 pulmonary function tests were performed in this laboratory. The computer system not only gathers the data but generates billing information, generates reports that are printed immediately at the appropriate nursing division, and provides interpretation of the patient data. The system also provides management information for the laboratory, as well as quality-control information generated as part of the normal course of data gathering. Operation of this laboratory without the computer would be an almost impossible task at this point. The computer provides legible reporting and documentation in a much more timely fashion than what would have been possible with the usual manual methods. The Battelle report showed that the physicians felt that the blood gas interpretation was a particularly effective way of helping the physicians make decisions and diagnosis and adjust therapy. Most of the physicians think that the blood gas interpretations save them time, and they value the service. Physicians had high expectations of the computerized pulmonary/blood gas area and indicated that the system met their expectations $81 \%$ of the time. These highly positive evaluation ratings have stimulated further refinement of the computerized pulmonary and blood gas system.

\section{FUTURE ENHANCEMENT FOR HELP}

Future enhancement for the HELP system is focused in three areas. The first is a continued effort to add to the medical decision protocols that currently are operational on the system. This is a continuing research effort combining both the clinical data collection and research capabilities of the system in collaboration with clinical researchers to enhance the existing logic and develop new logic. The second area of focus is a 
continued effort to enhance the speed and throughput of the system. The major redesign in this area will be in distributing the data base and the programs throughout the hospital using "state of the art" microprocessor-based systems at each nursing division. In this design the system, while storing the information in its coded form at the central data base for complete HELP processing, will also transmit in a formated form all data and HELP decisions to the nursing divisions systems, where speed of review of this information is provided to the medical personnel at that location. This not only will increase the speed of review for individuals at the respective nursing divisions but, because of decreased load on the central system, will also increase the speed with which the HELP processing can be performed at the central system. The third area of enhancement will be to place microprocessor-based HELP systems in the doctor's office. These systems will be interfaced to the central data base. Then, through communication with central data base and a processing system of similar capabilities for analysis, data recorded within the doctor's office can be provided to users outside the hospital. This will allow continued data entry into the patient data base and follow-up of the patient from the doctor's office.

\section{BIBLIOGRAPHY}

1. Warner, H. R., Olnsted, C. M., and Rutherford, B. D., HELP-A program for medical decision-making. Comput. Biomed. Res. 5:65-74, 1972.

2. Warner, H. R., Rutherford, B. D., and Houtchins, B., A sequential bayesean approach to history taking and diagnosis. Comput. Biomed. Res. 5:256-262, 1972.

3. Pryor, T. A., Lindsay, A. E., and England, R. W., Computer analysis for serial electrocardiograms Compute. Biomed. Res. 5(6), 1972.

4. Pryor, T. A., Morgan, J. D., Clark, S., England, W., and Warner, H. R. HELP-A computer system for medical decision-making. Presented at National Computer Conference and Exposition, May 1974

5. Clayton, P. D., Urie, P. M., Marshall, H. W., and Warner, H. R., A computerized system for the cardiovascular laboratory. IEEE Proceedings of Conference on Computers in Cardiology, Bethesda, Maryland, October 1974, p. 97.

6. Yanowitz, F. G., Pryor, T. A., Morgan, J. D., and Warner, H. R., The HELP system for medical decision making. Proceedings of the 4th Annual Conference of the Society for Computer Medicine, New Orleans, November 7-10, 1974 (Michael A. Jenkin, ed.).

7. Gardner, R. M., Cannon, G. H., Morris, A. H., Olsen, K. R., and Price, G. A., Computerized blood gas interpretation and reporting system. IEEE Comput. 8:19, 1975.

8. Gardner, R. M. The place of computers in medicine. Guest editor's introduction. IEEE Comput. 8:3945,1975 .

9. Warner, H. R., Pryor, T. A., Morgan, J. D., and Clark, S. J. Integration of computer support for institutional practice using the HELP system. Proceedings of SAMS meeting, Houston, Texas, 1975.

10. Yanowitz, F. G., Pryor, T. A., and Frost, D., A computerized ECG alarm system for the coronary care unit. Computers in Cardiology (J. Cox and P. Hugenholtz, ed), St. Louis, 1976.

11. Hulse, R. K., Clark, S. J., Jackson, J. C., Warner, H. R., and Gardner, R. M. Computerized medication monitoring system. Am. J. Hosp. Pharm. 33:1061-1064, 1976.

12. Pryor, T. A., and Yanowitz, F. G., Computer applications to exercise stress testing. Computers in Cardiology (J. Cox and P. Hugenholtz, eds.), St. Louis, 1976.

13. Warner, H. R., Pryor, T. A., Clark, S., and Morgan, J., Integration of computer support for institutional practice: The HELP system. Comput. Appl. Health Care Delivery, 1976.

14. Frost, D. A., Yanowitz, F. G., and Pryor, T. A., Evaluation of a computerized arrhythmia alarm system. Am. J. Cardiol. 39:583-587, 1977. 
15. Gardner, R. M., Scoville, D. P., West, B. J., Cundick, R. M., Jr., and Clemmer, T. P. Integrated computer systems for monitoring of the critically ill. Proceedings First Annual Symposium on Computer Application in Medical Care, October 3-5, 1977, IEEE Computer Society, Washington, D.C. pp. 301-307.

16. Gardner, R. M., and Clemmer, T. P., Computerized protocols applied to acute patient care. Advances in Automated Analysis, Vol. 1, Mediad Inc. Technicon International Congress, New York., 1977, pp. 158-163.

17. Pryor, T. A., Ho, S. J., and Stephen, R. L., Computer system for management and research in end-stage renal disease. Curr. Topics Crit. Care Med. 3:94-111, 1977.

18. Warner, H. R., First the electrocardiogram-then what? Am. J. Cardiol. p:5-8, 1977.

19. Stephen, R. L., Pryor, T. A., Jacobsen, S. C., Janata, J., and Kablitz, C., A computerized closed-loop feedback control of treatment for patients on dialysis. MECO '78-Athens, Greece, June 26-29, 1978.

20. Pryor, T. A., Gardner, R. M., Clayton, P. D., and Warner, H. R., A distribute processing system for patient management. Proc. Comput. Cardiol. p:325-328, September 1978.

21. Gardner, R. M., and Clemmer, T. P., Computerized protocols applied to Emergency and acute care. Emergency Med. Serv. 7:90-93, 1978.

22. Gardner, R. M., Clemmer, T. P., Larsen, K. G., and Johnson, D. S., Computerized alert system use in clinical medicine. IEEE 1979 Frontiers of Engineering in Health Care. First Annual Conference, Denver, October 1979, pp. 136-140.

23. Warner, H. R., Computer-Assisted Medical Decision-Making. Book published by Academic Press, New York, 1979.

24. Warner, H. R., Clark, S. J., Larsen, K. G., and McNeil, F., The HELP system as a tool for monitoring physician prescribing patterns. Proceedings of the 13th Hawaii International Conference on System Sciences, Vol. 3 (Shriver and Sprague, eds.), Western Periodicals, 1980.

25. Warner, H. R., Computer-assisted decision-making using clinical and paraclinical (laboratory) data. Minnesota Symposium on Decision-Making from Laboratory Data, University of Minnesota Press, Minneapolis, 1980 .

26. Cundick, R. M., Jr., and Gardner, R. M., Clinical comparison of pressure pulse and indicator dilution cardiac output determinations. Circulation, 62:371-376, 1980.

27. Pryor, T. A., Warner, H. R., and Gardner, R. M., HELP-A total hospital information system. Proceedings of the 4th Annual Symposium on Computer Application in Medicine Care, (Joseph T. O'Neill, ed.), Washington, D.C., November 2-5, 1980, pp. 1557-1561.

28. Warner, H. R., HELP-An approach to hospital-wide artificial intelligence. Computer-Assisted Decision Making Using Clinical and Paraclinical (Laboratory) Data, (Gernard E. Statland and Stanley Bauer, eds.), Mediad, Tarrytown, New York, 1980.

29. Johnson, D. S., Ranzenberger, J., Herbert, R. D., Gardner, R. M., and Clemmer, T. P., Computerized alarm system for acutely ill patients. J. Nurse Admin. 10:26-35, 1980.

30. Cannon, S. R., and Gardner, R. M., Experience with a computerized interactive protocol system using HELP. Comput. Biomed. Res. 13:399-409, 1980.

31. Sorensen, J. B., Morris, A. H., Crapo, R. O., and Gardner, R. M., Selection of the best spirometric values for interpretation. Am. Rev. Respir. Dis. 122:802-805, 1980.

32. Clemmer, T. P., Gardner, R. M., and Orme, J. R., Jr., Computer support in critical care medicine. IEEE Proceedings 4th Annual Symposium on Computer Applications in Medical Care, 1980, pp. 1557-1561.

33. Clayton, P. D., Ostler, D. B., Gennaro, J. L., Beatty, S. S., and Frederick, P. R., A radiology reporting system based on most likely diagnoses. Comput. Biomed. Res. 13:258-270, 1980.

34. Pryor, T. A., Clayton, P. D., Gardner, R. M., Waki, R., and Warner, H. R., HELP-A hospital-wide system for computer-based support of decision-making. Proceedings of the 14th Annual Hawaii International Conference on Systems Sciences, January 8, 1981.

35. Crapo, R. O., Gardner, R. M., and Beus, M. L., Lower limits of normal for spirometric reference values 1981 Conference of Computers in Critical Care and Pulmonary Medicine, Norwalk, Conn., in press.

36. Gardner, R. M., Ostler, D. V., and Crapo, R. O., Signal processing for computerized spirometry. 1981 Conference of Computers in Critical Care and Pulmonary Medicine, Norwalk, Conn., in press.

37. Waki, R., Clayton, P. D., Jensen, R. L., Yanowitz, F. G., and Liddle, H. V., Patient specific medical decision analysis using the HELP system. Proceedings of the Fifth Annual Symposium on Computer Applications in Medical Care (H. G. Heffernan, ed.), IEEE Computer Society Press, 1981, p. 237. 
38. Clayton, P. D., Gray, S., and Frederick, P. R., A code oriented radiology reporting system based upon pre-examination prediction of likely findings. Proceedings of the American College of Radiology Seventh Conference on Computer Applications in Radiology, April 1982, pp. 261-272.

39. Pryor, T. A., Clayton, P. D., and Larsen, K. L., An integrated hospital information system. Comput. Hosp., November 1982.

40. Pryor, T. A., Clayton, P. D., and Larsen, K. G., Synergistic relationship between test ordering and medical decision-making in the HELP hospital information system. Computers in Hospitals, Vol. 3, 6:36$40,1982$.

41. Pryor, T. A., Gardner, R. M., Clayton, P. D., and Warner, H. R., The HELP system. Proceedings of the 6th Annual Symposium of Computer Applications in Medical Care (H. G. Heffernan, ed.), IEEE Computer Society Press, 1982.

42. Waki, R., Clayton, P. D., Jensen, F. L., Yanowitz, F. G., and Liddle, H. V., HELP based decision analysis applied to coronary artery disease. Comput. Biomed. Res. 15:188-202, 1982.

43. Neeley, J. P., Ostler, D. B., Frederick, P. R., and Clayton, P. D., Pre-examination prediction of radiographic findings. Invest. Radiol. 17:310-315, 1982.

44. Gardner, R. M., West, B. J., Pryor, T. A., Larsen, K. G., Warner, H. R., Orme, J. F., Jr., and Clemmer, T. P., Computerized decision-making applied to critical care. Crit. Care Med. 10:823-830, 1982.

45. Gardner, R. M., Crapo, R. O., and Morris, A. H., Computerized decision-making in the pulmonary function laboratory. Resp. Care 27:799-808, 1982.

46. Morris, A. H., Kanner, R. E., Crapo, R. O., and Gardner, R. M., Clinical Pulmonary Function Testing: A Manual of Uniform Laboratory Procedures for the Intermountain Area. 2nd ed. 1983.

47. Gardner, R. M., Clemmer, T. P., and Morris, A. H., Computerized medical decision-making-An evaluation in acute care. Computers in Critical Care and Pulmonary Medicine, Vol. 2 (O. Prakash, ed.), Plenum Press, New York, 1982, pp. 147-150. 